\title{
¿Qué ha pasado con la aplicación del estudio de tiempos y movimientos en las últimas dos décadas?: Revisión de la literatura
}

\section{What happened with the application of time and motion study in the last two decades?: review of the literature}

\author{
Alex Mauricio Ovalle-Castiblanco ${ }^{1}$, Diana María Cárdenas Aguirre ${ }^{2}$ \\ ${ }^{1}$ Universidad Autónoma de Manizales, (Manizales-Caldas, Colombia). Grupo de diseño mecánico y desarro- \\ Ilo industrial, \\ movalle@autonoma.edu.co \\ ${ }^{2}$ Universidad Nacional de Colombia, (Manizales-Caldas, Colombia). Grupo de innovación y desarrollo, \\ dmcardenasa@unal.edu.co
}

(Recibido marzo 12 de 2016 y aceptado mayo 24 de 2016)

\begin{abstract}
Resumen
El estudio de tiempos y movimientos es una técnica de la ingeniería industrial que busca aumentar la productividad de las organizaciones, eliminando en forma sistemática las operaciones que no agregan valor al proceso y se constituye en la base para la estandarización de los tiempos de operación.

El presente trabajo pretende identificar la aplicación que el estudio de tiempos y movimientos ha tenido durante las últimas dos décadas, así como las técnicas y herramientas más utilizadas y los sectores a los que ha sido aplicado; para lo cual fueron consultadas las bases de datos Science Direct y Web of Science y se utilizó la herramienta tecnológica de análisis de redes de citaciones denominada tree of science, desarrollada en la Universidad Nacional de Colombia sede Manizales. A partir del análisis se pudo determinar que el estudio de tiempos con cronómetro, aplicado en su forma tradicional, es la técnica más utilizada para la medición de las tareas, adicionalmente se presenta una notoria tendencia a la realización de investigaciones en el sector salud.
\end{abstract}

Palabras clave: estudio de tiempos y movimientos, tree of science, técnicas de medición

\begin{abstract}
The time and motion study is an industrial engineering technique that seeks to increase the productivity of organizations, systematically eliminating operations that do not add value to the process and constitutes the basis for the standardization of operating times.

This paper aims to identify the application that the time and motion study has had over the past two decades, as well as techniques and tools most used and the sectors to which has been applied. For which they were consulted databases and direct science web of science and technology network analysis tool called citation tree of science, developed at the National University of Colombia in Manizales was used. From the analysis it was determined that the time study with stopwatch, applied in its traditional form, is most often used for measuring tasks technique, additionally a marked tendency to conducting presents research in the health sector.
\end{abstract}

Key words: time and motion study, tree of science, measurement techniques.

Cómo citar este artículo:

Ovalle-Castiblanco, A.M., \& Cárdenas-Aguirre, D.M. (2016). ¿Qué ha pasado con la aplicación del estudio de tiempos y movimientos en las últimas dos décadas?: revisión de la literatura. Revista Ingeniería Investigación y Desarrollo, 16 (2), pp. 12-31 


\section{INTRODUCCIÓN}

El entorno global ha llevado a las organizaciones a buscar la mejora de sus procesos por medio de la identificación y eliminación en forma gradual de las actividades que no generan valor a sus productos y procesos. Estas actividades representan costos operacionales que se traducen en despilfarros de tiempo, materiales, espacio y demás recursos organizacionales.

Una de las técnicas más utilizadas para superar dichas deficiencias y elevar la productividad de los trabajadores es el estudio del trabajo, definido como el examen sistemático de los métodos para realizar actividades con el fin de mejorar la utilización eficaz de los recursos y de establecer normas de rendimiento con respecto a las actividades que se están realizando (Kanawaty, 1996), tuvo sus orígenes a principios del siglo XX, con los trabajos realizados por Frederick W. Taylor y continuados unos años después por los esposos Gilbreth (Baumgart \& Neuhauser, 2009; Lopetegui et al., 2014; Nor, 2008; Duran, 2007).

Estas técnicas logran definir las mejores formas para realizar las operaciones y el tiempo requerido para completar una tarea a una velocidad o ritmo normal y en condiciones determinadas (Al-Saleh, 2011; Meyers, 2005; Turner, 1993); aplican los conceptos de simplificación y los principios de economía de movimientos básicos para el diseño de operaciones, (Mizuyama, Yamada, Tanaka, \& Maki, 2013), ayudan a la eliminación gradual de las operaciones que no agregan valor a los productos, proporcionan elementos de medición del desempeño, diseñan métodos y procedimientos que contribuyen a la satisfacción de los trabajadores y su productividad especialmente en tareas repetitivas (Barnes, 1980; Puvanasvaran, 2013).

El estudio de movimiento implica el análisis de los movimientos básicos de la mano, brazos y cuerpo, utilizados para llevar a cabo una tarea. Incluye el diseño del lugar de trabajo, el medio ambiente, así como las herramientas y el equipo utilizado en la operación (por ejemplo; portapiezas, accesorios, herramientas manuales y máquinas herramientas) (Groover, 2007). Para Niebel \& Freivalds (2004), el estudio de métodos o movimientos, incluye el diseño, creación y selección de los mejores métodos, procesos, herramientas, equipos y habilidades de manufactura para fabricar un producto, adicionalmente deben considerar sobre cualquier cosa la seguridad de los trabajadores (Meyers, 2005).

Por su parte, la medición del trabajo establece el tiempo para llevar a cabo una tarea por trabajador, con base en un número limitado de observaciones (García, 2005). Autores como Karger \& Bayha, (1987); Kanawaty, (1996); Yusoff, Jaffar, Abbas \& Saad, (2012); Best, (2010); Adam \& Ebert (1991); Caso(2006), coinciden en afirmar que la medición del trabajo proporciona un medio para medir el tiempo empleado en la realización de una operación o una serie de operaciones de tal manera que separa los tiempos efectivos de los que no lo son; la figura 1 consolida la forma como la medición del trabajo es realizada a través de las técnicas del muestreo de trabajo, estudio de tiempos con cronómetro, estándares de tiempo predeterminado y datos estándar (Kanawaty, 1996; Baines, 1995).

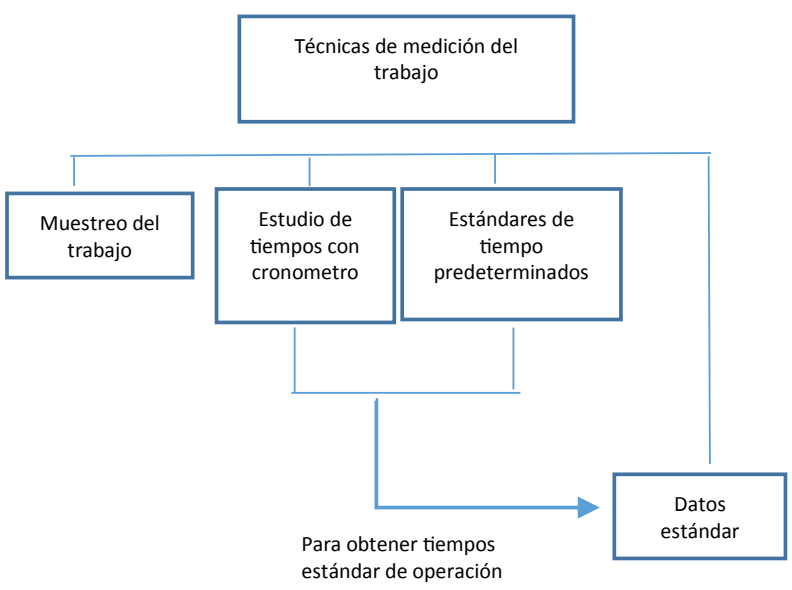

Figura 1.Técnicas de la medición del trabajo. Fuente: Adaptada de Kanawaty (1996)

El muestreo del trabajo es una de las técnicas más utilizadas en la medición del trabajo, consiste en la observación de una muestra o parte de la actividad laboral, y con referencia a ésta, determinar resultados respecto a toda la actividad (Chase, Jacobs \& Aquilano, 2009). Para Russell \& Taylor (2003), el muestreo de trabajo determina la proporción de tiempo que un trabajador pasa en las actividades, y relaciona porcentualmente los retardos o inactividades de máquinas y personas.

Según (Aft, 2000) el estudio de tiempo, con cronómetro, es el tipo más antiguo de la medición del trabajo, mide el tiempo que tarda un trabajador medio para

Rev. 12+D. Vol.16 N². Julio-Diciembre. 2016. Sogamoso-Boyacá, Colombia. 
completar una tarea a un ritmo normal y de manera repetitiva, (Nakayama, Nakayama, \& Nakayama, 2002 , Vonderembse \& White ,2003, Hodson, 1996); hoy en día , además de las herramientas estándar de estudio de tiempos con cronómetro, se utilizan ordenadores, cámaras de video, códigos de barras y softwares especializados (Izetbegovic , 2007,citado por Tanner ,1990).

Autores como Nor (2008), Garcia (2005); Lee \& Chan (2003), definen los estándares de tiempos predeterminados como el análisis de cualquier actividad manual en términos de movimientos básicos o fundamentales necesarios para su realización, a los cuales se le asigna un valor de tiempo estándar que ha sido previamente establecido. Éstos son el resultado de estudiar una gran muestra de operaciones diversificadas con un dispositivo de medición de tiempo, como una cámara de cine o de video grabación capaz de medir lapsos muy pequeños de tiempo (Wygant, 1989). El procedimiento consiste en dividir las tareas en elementos o micromovimientos y medirlos en una unidad de tiempo que se denominan UTM (unidad de medida de tiempos) cuya conversión es 1UMT = 0.00001 hora $=1 / 1000.000$ hora $=0.0006$ minutos $=0.036$ segundos.

El objetivo de este trabajo es presentar una revisión de la literatura de los artículos que se han publicado en las últimas dos décadas, en el campo del estudio de tiempos y movimientos, apoyados por dos herramientas de búsqueda: La primera denominada el árbol de la ciencia (ToS, por sus siglas en inglés), que realiza una clasificación de la literatura por medio de un análisis de redes de citaciones (Robledo, Osorio \& Lopez, 2015) y la segunda es la base de datos multidisciplinar Science Direct, perteneciente al grupo Elsevier. Con el fin de identificar cuáles son las herramientas más utilizadas dentro del campo de los tiempos y movimientos, el trabajo se encuentra organizado según las cuatro técnicas principales de la medición del trabajo: Estudio de tiempos con cronómetro, muestreo del trabajo, estándares de tiempos predeterminados y datos estándar, finalmente se presentan las conclusiones, las cuales servirán de base para determinan las tendencias y futuros trabajos de investigación.

\section{METOdOLOGÍA}

Para realizar la revisión de la literatura se definió una ecuación de búsqueda basada en los siguientes criterios:

Como primer criterio se utilizó la base de datos Science Direct, (buscador multidisciplinar), haciendo uso de la siguiente ecuación de búsqueda: Tema (time and motion study) en el título o en el abstract, entre los años 1996 y 2016, arrojando como resultado 19 artículos.

El segundo criterio fue la utilización de la base de datos Web of Science (WOS) desde enero de 1996 hasta febrero de 2016, con la ecuación de búsqueda topic: ("time motion studies") or topic: ("time and motion study") or topic: ("time-motion study"), en el título/abstract, encontrando 249 resultados los cuales son incorporados a la herramienta de búsqueda desarrollada por investigadores de la Universidad Nacional de Colombia sede Manizales ,el Tree of Science (ToS), que consiste en un análisis de redes de citaciones a partir de la teoría de grafos, clasificando en forma gráfica de árbol los artículos relacionados (Robledo, Osorio \& López, 2015). El árbol encontrado consta de 79 artículos distribuidos de la siguiente manera: los artículos que empezaron el área de investigación, están determinados por los puntos color naranja y representan las raíces (10 artículos), en el tronco se ubican los artículos que citan y a la vez son más citados dentro del área de estudio, representados por puntos color café ( 9 artículos) y finalmente en las hojas, los artículos más recientes sobre la temática de investigación y que por lo tanto aun no son muy citados, puntos verdes (60 artículos).ver figura 2.

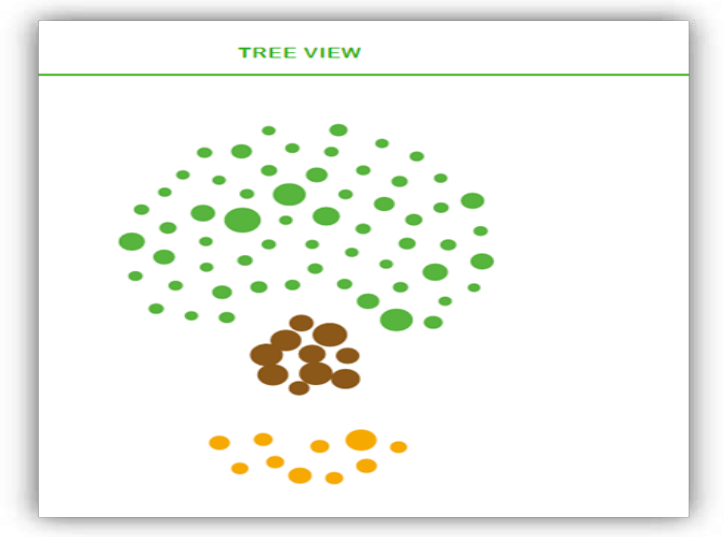

Figura 2. Árbol de la ciencia del proceso de búsqueda. Fuente: Robledo, Osorio \& López (2015). 
La figura 3 ilustra como la herramienta codifica los datos de forma tal que al hacer "click" en el eslabón de color se redirecciona hacia el artículo referenciado.
Por último se cruzaron los datos arrojados por las dos bases de datos, encontrándose 8 artículos que se encuentran simultáneamente en las dos bases de datos lo que determina un total de 90 artículos que cumplen con los criterios de selección y pertinencia del tema.
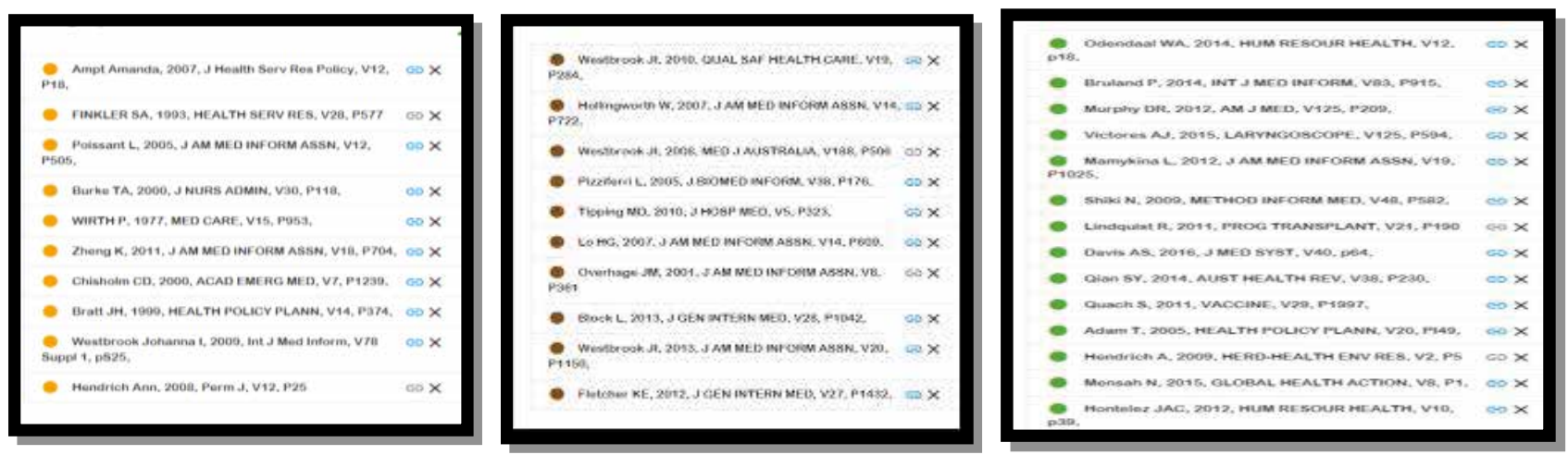

Figura 3. Codificación de la información arrojada por el ToS.

Fuente: Robledo, Osorio \& López (2015)

\section{RESULTADOS}

\subsection{Estudio de tiempos con cronómetro}

El $89,5 \%$ de los artículos estudiados utilizaron la técnica del estudio de tiempos con cronómetro (tablas 1,2 y 3 ), con algunas variaciones en su aplicación, complementándose con elementos adicionales tales como: el uso de cámaras de video, observación directa, análisis de datos a través de software especializado, entrevistas y encuestas de satisfacción, así como la comparación de datos de cronómetro con otros medios electrónicos de recolección de información.
En la tabla 1 se puede apreciar que el $7 \%$ de los proyectos utilizaron las cámaras de video para recoger la información y luego analizar los datos identificando los movimientos realizados por las personas y el tiempo invertido en la realización de las tareas (artículos 1-6), el artículo 1 por ejemplo hace un análisis de los videos de las sesiones de reanimaciones cardiopulmonar (RCP) entre diciembre de 2005 y diciembre de 2008 en el servicio de urgencias de un hospital y mediante un análisis de tiempo-movimiento, logran determinar que se invierte un tiempo significativo en actividades de despliegue médico y que la falta de familiaridad con las estrategias de posicionamiento de los dispositivos necesarios repercuten en un bajo rendimiento en la tarea. 
Tabla 1.Estudio de tiempo con cronómetro (cámara de video-observación y software especializado-encuestas).

\begin{tabular}{|c|c|c|c|c|c|c|}
\hline Artículos & Año & Sector & $\begin{array}{l}\text { Técnica } \\
\text { utilizada }\end{array}$ & $\begin{array}{l}\text { Objetivo del } \\
\text { estudio }\end{array}$ & $\begin{array}{c}\text { Número de } \\
\text { artículos }\end{array}$ & $\%$ \\
\hline 1. Huang et al & 2013 & \multirow{5}{*}{ salud } & \multirow{6}{*}{$\begin{array}{l}\text { Estudio de } \\
\text { tiempos con } \\
\text { cronometro- } \\
\text { Cámara de } \\
\text { video }\end{array}$} & \multirow{6}{*}{$\begin{array}{l}\text { Análisis de los } \\
\text { métodos y } \\
\text { tiempos a } \\
\text { través de la } \\
\text { reproducción } \\
\text { de los videos }\end{array}$} & \multirow{6}{*}{6} & \multirow{6}{*}{7} \\
\hline 2. Shabbir et al & 2010 & & & & & \\
\hline 3. Roman et al & 2016 & & & & & \\
\hline 4. Cady \& Finkelstein & 2014 & & & & & \\
\hline 5.Saurman et al & 2014 & & & & & \\
\hline 6. Rudkin \& O'Donoghue & 2008 & entretenimeinto & & & & \\
\hline 7. Westbrook \& Ampt & 2009 & \multirow{8}{*}{ salud } & \multirow{10}{*}{$\begin{array}{l}\text { Estudio de } \\
\text { tiempos con } \\
\text { cronometro- } \\
\text { observación y } \\
\text { software }\end{array}$} & \multirow{10}{*}{$\begin{array}{l}\text { analisis de } \\
\text { tiempos a } \\
\text { traves de } \\
\text { software } \\
\text { especializado }\end{array}$} & \multirow{10}{*}{10} & \multirow{10}{*}{11,5} \\
\hline 8. Burker et al & 2000 & & & & & \\
\hline 9.Tipping et al & 2010 & & & & & \\
\hline 10.Fletcher et al & 2012 & & & & & \\
\hline 11.Lo et al & 2007 & & & & & \\
\hline 12. Zheng et al & 2011 & & & & & \\
\hline 13. Day et al & 2014 & & & & & \\
\hline 14. Mensah et al & 2015 & & & & & \\
\hline 15. Tinoco et al & 2015 & educación & & & & \\
\hline 16.Carreira et al & 2013 & agricola & & & & \\
\hline 58. Thorpe-Jamison et al & 2013 & \multirow{7}{*}{ salud } & \multirow{7}{*}{$\begin{array}{l}\text { Estudio de } \\
\text { tiempos con } \\
\text { cronometro- } \\
\text { observacion- } \\
\text { cronometro- } \\
\text { encuesta }\end{array}$} & \multirow{7}{*}{$\begin{array}{c}\text { Tiempo } \\
\text { dedicado a las } \\
\text { tareas, manera } \\
\text { de hacerlas } \\
\text {,entrevista y } \\
\text { encuesta de } \\
\text { satisfacción }\end{array}$} & \multirow{7}{*}{7} & \multirow{7}{*}{7,5} \\
\hline 59. Rothberg et al & 2012 & & & & & \\
\hline 60.Philpott et al & 2013 & & & & & \\
\hline 61.Wong \& Bradley & 2009 & & & & & \\
\hline 62.Rudking et al & 2006 & & & & & \\
\hline 63.Asfaw et al & 2014 & & & & & \\
\hline 64.Manzi et al & 2012 & & & & & \\
\hline
\end{tabular}

Fuente: Propia

Por su parte un $11,5 \%$ de los trabajos aplicó o desarrollo software para la recolección y análisis de la información de los tiempos y movimientos necesarios para cada la tarea (artículos del 7-16)

Un ejemplo de la técnica se encuentra en el estudio 15 , que propone un enfoque para llevar a cabo análisis de tiempo y movimiento con un sistema de captura de movimiento y entornos virtuales, usando pistas de marcadores pasivos infrarrojos situados en las manos para obtener las posiciones de cada mano.
En la misma tabla 1, se puede observar que el 7,5\% de las investigaciones aplicaron la observación directa-toma de tiempos con cronometro entrevistas y encuestas de satisfacción (artículos del 58-64), el artículo 59 por ejemplo, realizó un estudio de tiempos con cronómetro al tiempo empleado por los médicos y las enfermeras en la atención a los pacientes, se encontró que en promedio, los médicos pasaron más tiempo por paciente en procesos de documentación de sus labores, que en la comunicación verbal con el paciente $(9.2 \mathrm{~min}$ vs $6,3 \mathrm{~min}$ ) 
La tabla 2 muestra que el mayor porcentaje de artículos (46\%), utilizaron la técnica del estudio de tiempos con cronómetro complementado con observación directa, con el objetivo de determinar el tiempo que las personas observadas invierten en las actividades o tareas previamente seleccionadas y de qué manera las realizan,(artículos del 17-57).

En el artículo 20 se realizó un estudio de tiempo y movimiento y mediante la observación directa, se hizo el seguimiento a enfermeras de la unidad de cuidados intensivos durante el turno de día, de lunes a viernes. Todas las actividades realizadas por las enfermeras durante su turno fueron cronometradas, registradas y codificadas según se trató de actividades que involucraron directa o indirecta a los pacientes; Este estudio proporcionó evidencia de base sobre las actividades que las enfermeras llevan a cabo diariamente, encontrándose que alrededor de una cuarta parte de su tiempo, no se gasta en actividades de atención al paciente, ya sea directa o indirectamente.

En la tabla 3 el 17,5\% de los artículos aplicaron la técnica del cronómetro para la medición de tareas y comparación de datos con elementos electrónicos y automáticos (artículos del 65-80) el artículo 74 es un claro ejemplo de este tipo de trabajos, a pesar de los beneficios asociados con el uso de registros electrónicos de salud (EHR), un importante obstáculo para su adopción es la preocupación de que los EHR puede tomar más tiempo para los médicos en su diligenciamiento que los sistemas basados en papel, para abordar este interrogante, se realizó un estudio de tiempo-movimiento en centros de atención primaria y se compararon los resultados utilizando las dos técnicas; el estudio reveló que aunque la mayoría considera que el sistema electrónico mejora la calidad, un gran porcentaje de médicos todavía no encuentra una notoria diferencia.

\subsection{Muestreo del trabajo y Estándares de tiempos pre- determinados}

Un caso de aplicación de la técnica de disposición modular de están dares de tiempos predeterminados (MODAPST) por sus siglas en inglés (artículo 81), fue encontrado en la revisión de la literatura; ver tabla 3 , dicha técnica consiste en un sistema de tiempo predeterminado que se ocupa de los valores de tiempo estándar o unidades de trabajo físico humano, denominados "módulos" o "mods". El trabajo consistió en la utilización de la herramienta MODAPST y la integración de la tecnología de análisis de movimiento, lo que ayudaría a hacer MODAPTS más eficiente y confiable, este av a nce permite un progreso significativo para el análisis de los factores humanos. 
Tabla 2. Estudio de tiempo con cronómetro (observación directa).

\begin{tabular}{|c|c|c|c|c|c|c|}
\hline Artículos & Año & Sector & $\begin{array}{l}\text { Técnica } \\
\text { utilizada }\end{array}$ & $\begin{array}{l}\text { Objetivo del } \\
\text { estudio }\end{array}$ & $\begin{array}{l}\text { Número de } \\
\text { artículos }\end{array}$ & $\%$ \\
\hline 17. Tuinman et al & 2015 & \multirow{39}{*}{ salud } & \multirow{41}{*}{$\begin{array}{l}\text { Estudio de } \\
\text { tiempos con } \\
\text { cronometro- } \\
\text { observacion y } \\
\text { cronometro }\end{array}$} & \multirow{41}{*}{$\begin{array}{c}\text { tiempo } \\
\text { dedicado a las } \\
\text { tareas y } \\
\text { manera de } \\
\text { hacerlas }\end{array}$} & \multirow{41}{*}{41} & \multirow{41}{*}{46} \\
\hline 18.De Coster et al & 2015 & & & & & \\
\hline 19.Prenner et al & 2015 & & & & & \\
\hline 20.Abbey et al & 2012 & & & & & \\
\hline 21.Mallidou et al & 2013 & & & & & \\
\hline 22.Wiedenmayer et al & 2009 & & & & & \\
\hline 23. Bratt et al & 1999 & & & & & \\
\hline 24. Chisholm et al & 2000 & & & & & \\
\hline 25.Westbrook et al & 2010 & & & & & \\
\hline 26. Overhage et al & 2001 & & & & & \\
\hline 27.Kee et al & 2012 & & & & & \\
\hline 28.Qian et al & 2012 & & & & & \\
\hline 29.Weigl et al & 2013 & & & & & \\
\hline 30. Mache et al & 2012 & & & & & \\
\hline 31.Qian et al & 2014 & & & & & \\
\hline 32. Weigl et al & 2009 & & & & & \\
\hline 33.Kreulen et al & 2000 & & & & & \\
\hline 34.Block et al & 2013 & & & & & \\
\hline 35. Kukafka et al & 2013 & & & & & \\
\hline 36.Suh et al & 2010 & & & & & \\
\hline 37.De Cock et al & 2015 & & & & & \\
\hline 38.Starmer et al & 2015 & & & & & \\
\hline 39.Wanyenze et al & 2010 & & & & & \\
\hline 40.Deo et al & 2012 & & & & & \\
\hline 41.Milosavljevic et al & 2011 & & & & & \\
\hline 42.Dwibedi et al & 2011 & & & & & \\
\hline 43. López et al & 2010 & & & & & \\
\hline 44.Van Oostveen et al & 2013 & & & & & \\
\hline 45. Odendaal \& Lewin & 2014 & & & & & \\
\hline 46. Arabadzhiyska et al & 2013 & & & & & \\
\hline 47.Chu \& Huang & 2013 & & & & & \\
\hline 48.Denton et al & 2005 & & & & & \\
\hline 49. Adam et al & 2005 & & & & & \\
\hline 50.Li et al & 2015 & & & & & \\
\hline 51.Mangham-Jefferies et al & 2014 & & & & & \\
\hline 52.Bruland et al & 2014 & & & & & \\
\hline 53. Hendrich et al & 2009 & & & & & \\
\hline 54. Hontelez et al & 2012 & & & & & \\
\hline 55.Shinder et al & 2012 & & & & & \\
\hline 56.Duran & 2007 & servicios & & & & \\
\hline 57.Spinelli \& Visser-Rien & 2009 & agricola & & & & \\
\hline
\end{tabular}

Fuente: Propia

Rev. 12+D. Vol.16 N². Julio-Diciembre. 2016. Sogamoso-Boyacá, Colombia. 
La tabla 3 también reúne la información de los artículos que aplicaron la técnica del muestreo del trabajo como técnica base para lograr la captura de la información (artículos del 82 - 86) y que equivalen a un 5,5\% del total. En el artículo 84 se escogieron tres especialidades médicas y por medio de una muestra representativa de pacientes que ingresaban a las salas clínicas en hospitales universitarios de Holanda se realizó un estudio de tiempos y movimientos, registrando el tiempo dedicado por los médicos y enfermeras en la atención al paciente. También los costos involucrados en la atención médica y de enfermería, quirúrgicos, intervenciones y procedimientos de diagnóstico, así como una estimación de la demanda de servicios de atención hospitalaria por paciente hospitalizado, finalmente se aplicó un análisis de regresión lineal para determinar los factores importantes, como las características del paciente y de los resultados de la salud.

En la misma tabla 3, se referencian tres estudios que realizaron revisión sistemática de la literatura en el campo de la salud sobre el tema de tiempos y movimientos, el artículo 89 enfocó la revisión a la estandarización de conceptos, la revisión reveló un malentendido común y recurrente en cuanto a la definición y el alcance de los estudios de tiempos y movimientos ya que el término, está siendo utilizado de dos maneras en la literatura biomédica, especialmente en el sector de la salud, en un nivel global se refiere al conglomerado de estudios en los que la duración de un evento es una de las variables de interés, y en un nivel más detallado, lo que hace referencia al uso de un tiempo de grabación de datos de un observador externo en forma continua.

La ecuación de búsqueda utilizada por los investigadores fue la siguiente: Se seleccionó la base de datos PubMed/ MEDLINE, se utilizó la siguiente ecuación de búsqueda(( "Time and motion studies"(Majr) o "time and motion study" (title/abstract) o "time motion study" (title/ abstract) AND "health care category(mesh) AND "ingles"(lang) AND "2003/01/01"(PDAT): "2013/01/01" (PDAT)AND Journal article(ptyp)NOT Editorial(ptyp)NOT review(ptyp) NOT comment(ptyp)). Encontrándose 263 artículos. Los autores recomiendan utilizar el concepto de tiempos y movimientos como:"observación continua de los movimientos y tiempos para capturar la información, independiente de la herramienta de captura".

Para la segunda revisión de la literatura encontrada (artículo 87), los investigadores realizaron una revisión sistemática de la literatura para examinar el impacto de los registros electrónicos de salud (EHR), por sus siglas en inglés, sobre el tiempo de la documentación de los médicos y las enfermeras e identificar los factores que pueden explicar las diferencias de eficiencia entre los estudios. En total, 23 artículos cumplieron los criterios de inclusión; cinco eran ensayos controlados aleatoriamente, seis eran estudios de control después de la prueba, y 12 eran de un grupo de diseño pretest -postest. 
Tabla 3. Estudio de tiempo con cronómetro, estándar de tiempo predeterminado y muestreo del trabajo

\begin{tabular}{|c|c|c|c|c|c|c|}
\hline Artículos & Año & Sector & Técnica utilizada & Objetivo del estudio & $\begin{array}{l}\text { Número de } \\
\text { artículos }\end{array}$ & $\%$ \\
\hline 65. Rebmann et al & 2009 & \multirow{15}{*}{ salud } & \multirow{16}{*}{$\begin{array}{l}\text { Estudio de } \\
\text { tiempos con } \\
\text { cronometro }\end{array}$} & \multirow{16}{*}{$\begin{array}{l}\text { Tiempo dedicado a } \\
\text { las tareas en forma } \\
\text { manual comparado } \\
\text { con medio } \\
\text { electrónico y/o } \\
\text { automático }\end{array}$} & \multirow{16}{*}{16} & \multirow{16}{*}{17,5} \\
\hline 66. Quach et al & 2011 & & & & & \\
\hline 67. Hollingworth et al & 2007 & & & & & \\
\hline 68.Westbrook et al & 2013 & & & & & \\
\hline 69.Murphy et al & 2012 & & & & & \\
\hline 70.Schofield et al & 2015 & & & & & \\
\hline 71.Mamykina et al & 2012 & & & & & \\
\hline 72.Ponn et al & 2008 & & & & & \\
\hline 73.Victores et al & 2015 & & & & & \\
\hline 74.Pizziferri et al & 2005 & & & & & \\
\hline 75.Davis et al & 2016 & & & & & \\
\hline 76.Were et al & 2010 & & & & & \\
\hline 77.Shabbir et al & 2010 & & & & & \\
\hline 78.Body et al & 2015 & & & & & \\
\hline 79.MacMillan et al & 2013 & & & & & \\
\hline 80.Torres et al & 2010 & agricola & & & & \\
\hline 81.Shuang et al & 2016 & salud & $\begin{array}{l}\text { Estandares de } \\
\text { tiempos } \\
\text { predeterminados }\end{array}$ & $\begin{array}{c}\text { Arreglo Modular de } \\
\text { Tiempos Estándares } \\
\text { Predeterminados } \\
\text { (MODAPTS)Clasificaci } \\
\text { ón de los } \\
\text { movimientos del } \\
\text { cuerpo } \\
\end{array}$ & 1 & 1 \\
\hline 82.Ampt et al & 2007 & \multirow{5}{*}{ salud } & \multirow{5}{*}{$\begin{array}{l}\text { Muestreo del } \\
\text { trabajo }\end{array}$} & \multirow{5}{*}{$\begin{array}{c}\text { Estudio de muestreo } \\
\text { para lograr } \\
\text { generalizacion de las } \\
\text { tareas }\end{array}$} & \multirow{5}{*}{5} & \multirow{5}{*}{5,5} \\
\hline 83.Finkler et al & 1993 & & & & & \\
\hline 84.Van Oostveen et al & 2015 & & & & & \\
\hline 85. Meguerditchian et al & 2013 & & & & & \\
\hline 86. Kilpatrick & 2013 & & & & & \\
\hline 87.Poissant et al & 2005 & \multirow{3}{*}{ salud } & $\begin{array}{l}\text { Revision de la } \\
\text { literatura }\end{array}$ & $\begin{array}{l}\text { Impacto de los } \\
\text { registros electronicos } \\
\text { de salud EHR }\end{array}$ & \multirow{3}{*}{3} & \multirow{3}{*}{3} \\
\hline 88. Leafloor et al & 2015 & & $\begin{array}{l}\text { Revision de la } \\
\text { literatura }\end{array}$ & $\begin{array}{l}\text { Estudio de tiempos y } \\
\text { movimientos en } \\
\text { residentes de } \\
\text { medicina interna } \\
\end{array}$ & & \\
\hline 89.Lopetegui et al & 2014 & & $\begin{array}{l}\text { Revision de la } \\
\text { literatura }\end{array}$ & $\begin{array}{l}\text { Estandarizacion de } \\
\text { conceptos sobre } \\
\text { tiempos y } \\
\text { movimientos }\end{array}$ & & \\
\hline 90.Magu et al & 2016 & servicios & $\begin{array}{l}\text { Path process } \\
\text { chart }\end{array}$ & Nueva técnica & 1 & 1 \\
\hline
\end{tabular}

Fuente: Propia

Rev. I2+D. Vol.16 N². Julio-Diciembre. 2016. Sogamoso-Boyacá, Colombia. 
Una búsqueda extensa de la literatura a partir de 1966 hasta el 2004 se realizó utilizando MEDLINE , CINAHL , HEALTHSTAR, y las bases de datos de salud actual. Las estrategias de búsqueda incluyeron el Medical Subject Headings ( $\mathrm{MeSH}$ ) asociada con las palabras clave que reflejaban los EHR y flujo de trabajo . La estrategia de búsqueda en MEDLINE se basó en los siguientes términos: informática de la salud, registros electrónicos, sistemas de registro s médicos, informática médica, sistemas de información, registros computarizados de pacientes, flujo de trabajo, tiempo y movimiento, la ejecución de tareas y análisis, rediseño del trabajo, sólo se seleccionaron los documentos de texto completo en inglés o francés publicados en revistas y actas revisadas por pares para una revisión adicional. Se excluyeron los editoriales, cartas y documentos conceptuales, se concluye que la utilización de EHR hace más eficiente el tiempo de recolección de la información comparado con los medios tradicionales de papel.

Adicionalmente el artículo 88 tuvo como propósito revisar toda la bibliografía disponible relacionada con los estudios de ti empo-movimiento de residentes de medicina interna, y entender qué parte de su tiempo se repartirá a diversas categorías de tareas, sugeridas por el Consejo de acreditación para Educación médica para Graduados (ACGME). Se realizaron búsquedas de artículos en Inglés entre 1941 y abril de 2013 de informes de estudios de tiempo-movimiento de los residentes de medicina interna en rotación a través de un servicio de medicina general en bases de datos electrónicas, se concluye que la aplicación de las normas hora- deber ACGME, no tuvo un efecto significativo en el porcentaje de tiempo dedicado a tareas particulares. Hay informes contradictorios sobre cómo los cambios de hora deber han afectado a la seguridad del paciente.

En el artículo 90 se desarrolló la propuesta de una nueva técnica para el estudio de tiempos y movimientos llamada gráfi ca de ruta de proceso (path process chart), dich a técnica propone el estudio tanto de la ruta tomada por el trabajador es decir, los lugares de trabajo utilizados y su interrelación, así como el proceso de trabajo, se realizó en tres fases: Fase I, en la que se recogió información de 510 amas de casa en su trabajo rutinario de hogar, Fase II, en el que se desarrolló una nueva técnica llamada tabla de recorrido de proceso y la Fase III, en el que la técnica fue utilizada para llevar a cabo un estudio en profundidad de la actividad de preparació $n$ de alimentos en 50 cocinas urbanas de la India. La técnica podría generar datos importantes en cuanto a la forma en que los diferentes lugares de trabajo eran utilizados y como se relacionan entre sí. Los result ados del estudio permitirán diseñar cocinas ergonómicamente para las amas de casa de la India.

\subsection{Tendencia en la aplicación del estudio de tiempos y movimientos}

Aunque la revisión de la literatura denota una marcada tendencia a la aplicación de la técnica de estudio de tiempos co n cronómetro en su forma tradicional, se empiezan a abrir camino la utilización de elementos tecnológicos como soporte a la captura de información, así como el análisis de los datos recolectados con software especializados y la comparación de dichos estudios 


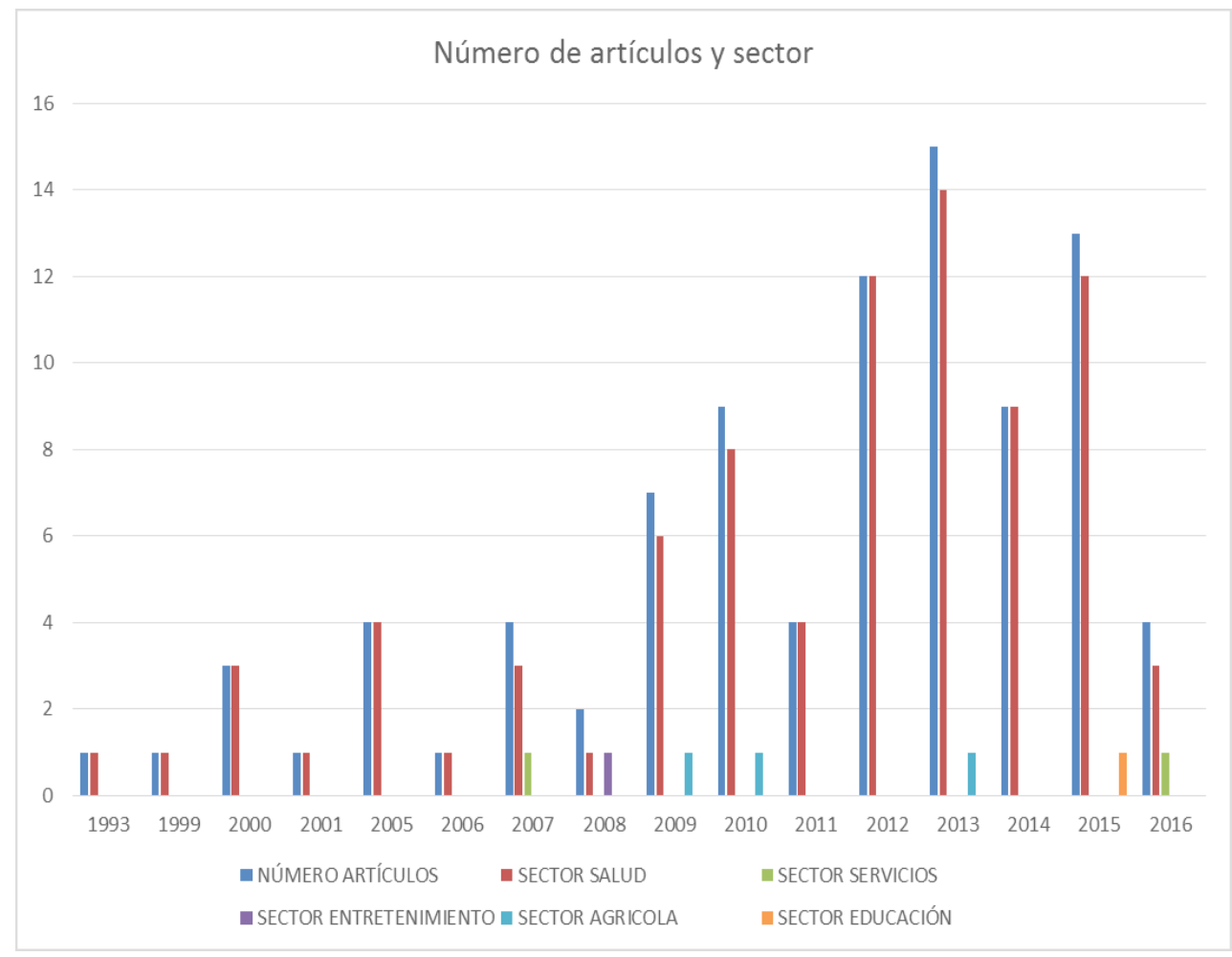

Figura 4. Número de artículos y sector por año.

Fuente: Propia

con medios electrónicos y automáticos para verificar la confiabilidad de los mismos, como lo demuestran los artículos del $1-16$ y del 65 al 80 y que representan un $35 \%$ de los artículos estudiados.

El sector salud es el más estudiado en el campo de referencia en los últimos 20 años, ya que de 90 artículos revisados en este estudio, 83 pertenecen a este sector, lo que equivale a un $92,2 \%$ de los artículos. En la figura 4 se representan los artículos realizados, el año de su publicación y el sector al que pertenece el estudio, aproximadamente el $74 \%$ de los proyectos fueron publicados en los últimos 6 años, demostrando así un creciente interés por parte de los investigadores sobre el tema de tiempos y movimientos y la aplicación en el sector salud por encima de otros sectores.

La ubicación geográfica de los 90 artículos estudiados muestra una concentración de los mismos en dos países, 32 artículos en Estados 


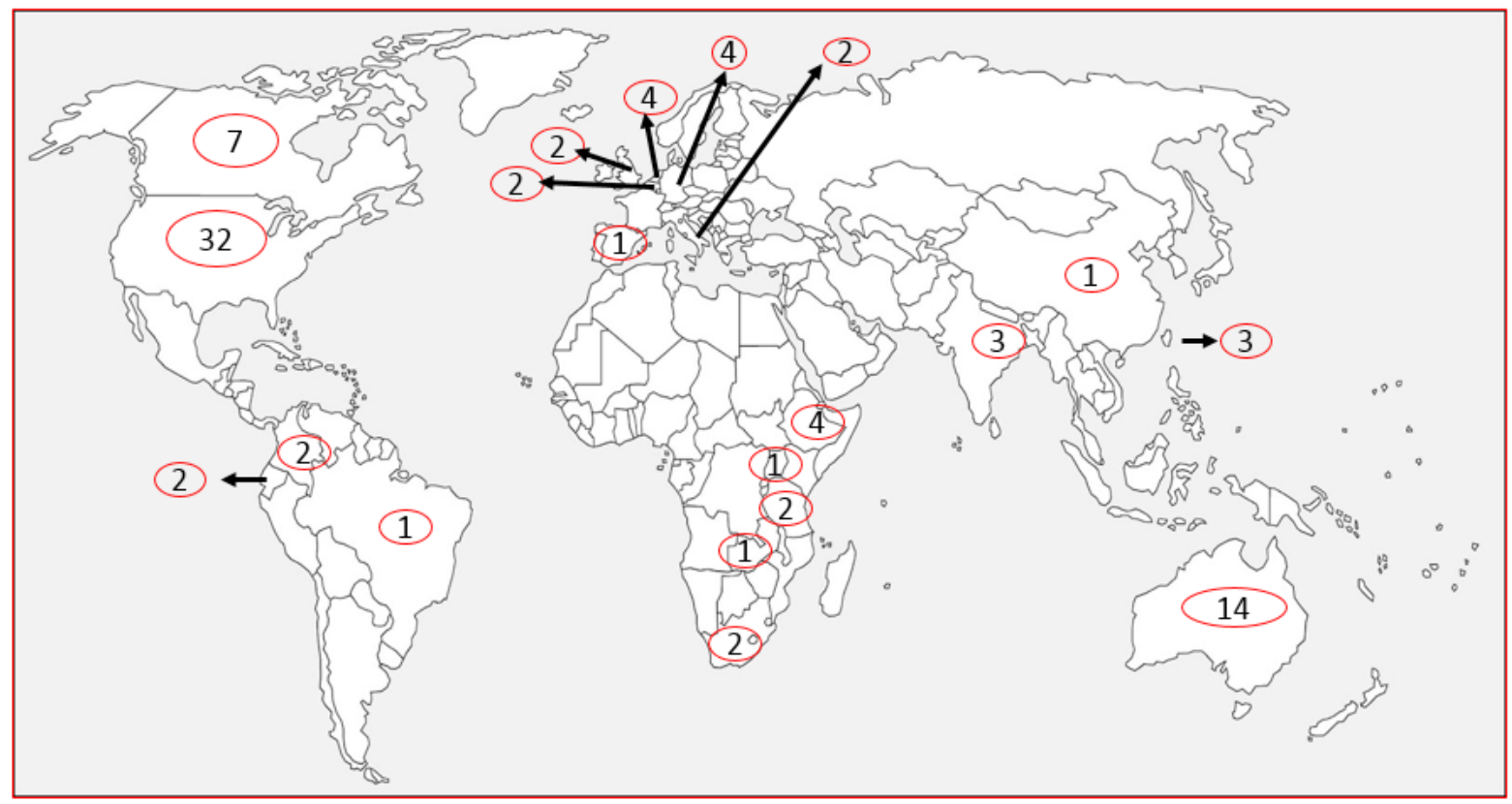

Figura 5. País y número de investigaciones

Fuente: Construcción propia a partir de imagen de commons.wikimedia.org.

Unidos y 14 en Australia, lo que equivale al $51 \%$ del total de las investigaciones, el restante número de proyectos se encuentra distribuido en diferentes países como se puede apreciar en la figura 5.

En el contexto latinoamericano vale la pena resaltar que sólo se encontraron 5 artículos, ubicados en Suramérica, dos pertenecen al sector salud, y los otros tres a los sectores servicios, educación y agricultura.

\section{CONCLUSIONES}

El estudio de tiempos con cronómetro de forma tradicional, representa la técnica más utilizada como elemento de medición de las tareas, encontrándose más del $89 \%$ de los trabajos desarrollados bajo ésta técnica.

La aplicación del estudio de tiempos y movimientos sigue teniendo vigencia en la actualidad, como lo demuestran las 66 investigaciones realizadas entre los años 2010 - 2016, las cuales aplicaron las técnicas de medición del trabajo en sus formas tradicionales de muestreo del trabajo, estudio de tiempos con cronómetro y estándares de tiempo predeterminados.
La utilización de la herramienta ToS, permite realizar una clasificación de la literatura por medio de un análisis de redes de citaciones, facilitando el trabajo de los investigadores a la hora de categorizar los artículos encontrados e indicando la presencia de una comunidad de investigadores que están enfocando sus esfuerzos sobre el campo de estudio de tiempos y movimientos y su aplicación en un contexto diferente al industrial como fue originalmente desarrollado.

Los estudios revisados sobre el uso de instrumentos de recolección de la información, como las cámaras digitales y software especializados para el análisis de los datos, buscan determinar una mayor confiabilidad en los datos obtenidos, eliminando la subjetividad del observador y minimizando el error humano en la captura de información cuando se utiliza la observación directa, como fue reportado en 16 de los 90 artículos estudiados.

El objeto de estudio en la relación paciente -atención, fue uno de los más encontrados en la revisión de la literatura, El $46 \%$ de los estudios de tiempos y movimientos permitieron determinar el tiempo que invierten los médicos y enfermeras en las actividades relacionadas con las atenciones a los pacientes y que

Rev. 12+D. Vol.16 N². Julio-Diciembre. 2016. Sogamoso-Boyacá, Colombia. 
porcentaje del mismo es utilizado en otras operaciones y labores.

Soportados en la revisión de la literatura se puede apreciar la falencia en los últimos años de investigaciones y revisiones que exploren otros campos y sectores de la economía, como por ejemplo el agrícola muy predominante y de gran importancia en el contexto latinoamericano.

\section{REFERENCIAS}

Abbey, M., Chaboyer, W. \& Mitchell, M. (2012). Understanding the work of intensive care nurses: a time and motion study. Australian Critical Care, 25(1), 13-22. DOI: http://dx.doi.org/10.1016/j. aucc.2011.08.002

Adam, E., \& Ebert, R. (1991). Administración de la producción y las operaciones: conceptos, modelos y funcionamiento. México: Pearson educación.

Adam, T., Amorim, D., Edwards, S., Amaral, J., \& Evans, D. (2005). Capacity constraints to the adoption of new interventions: consultation time and the Integrated Management of Childhood Illness in Brazil. Health policy and planning, 20(suppl 1), i49-i57. DOI: http://dx.doi.org/10.1093/heapol/czi057

Aft, L. (2000). Work measurement and methods improvement .Vol. 9. John Wiley \& Sons.

Al-Saleh, K. (2011). Productivity improvement of a motor vehicle inspection station using motion and time study techniques. Journal of King Saud University-Engineering Sciences, 23(1), 33-41. DOI: http://dx.doi.org/10.1016/j.jksues.20100.01.001

Ampt, A., Westbrook, J., Creswick, N., \& Mallock, N. (2007). A comparison of self-reported and observational work sampling techniques for measuring time in nursing tasks. Journal of health services research \& policy, 12(1), 18-24. DOI: http/ dx.doi.org/10.1258/135581907779497576

Arabadzhiyska, P., Baysari, M., Walter, S., Day, R., \& Westbrook, J. (2013). Shedding light on junior doctors' work practices after hours. Internal medicine journal, 43(12), 1321-1326. DOI: http://dx.doi.org/10.1111/imj.12223

Asfaw, E., Dominis, S., Palen, J., Wong, W., Bekele, A., Kebede, A., \& Johns, B. (2014). Patient satisfaction with task shifting of antiretroviral services in Ethiopia: implications for universal health coverage. Health policy and planning, 29(suppl 2), ii50-ii58. DOI: http://dx.doi.org/10.1093/ heapol/czu072

Baines, A. (1995). Workmeasurement-the basicprinciples revisited. Work Study, 44(7), 10-14. DOI: http:// dx.doi.org/10.1108/00438029510096553

Barnes, R. (1980). Motion and Time Study; Design and Measurement of Work (17 ed.). New York: John Wiley.

Baumgart, A., \& Neuhauser, D. (2009). Frank and Lillian Gilbreth: scientific management in the operating room. Quality and Safety in Health Care, 18(5), 413-415. DOI: http://dx.doi.org/10.1136/ qshc.2009.032409

Best, T. (2010). Work measurement in skilled labor environments. Retrieved August, 2, 2013.

Block, L., Habicht, R., Wu, A., Desai, S., Wang, K., Silva, K., ... \& Feldman, L. (2013). In the wake of the 2003 and 2011 duty hours regulations, how do internal medicine interns spend their time?. Journal of general internal medicine, 28(8), 1042-1047. DOI: http://dx.doi.org/10.1007/s11606-0132376-6

Body, J., Mebis, J., Peeters, M., Besse-Hammer, T., Fikkert, V., de Cock, E., ... \& Hechmati, G. (2015). A Time and Motion Study of Denosumab Subcutaneous Injection and Zoledronic Acid Intravenous Infusion in Patients with Metastatic Bone Disease in Belgium. Value in health: the journal of the International Society for Pharmacoeconomics and Outcomes Research, 18(7), A485.

Bratt, J., Foreit, J., Chen, P., West, C., Janowitz, B., \& De Vargas, T. (1999). A comparison of four approa- 
ches for measuring clinician time use. Health policy and planning, 14(4), 374-381. DOI: http://dx.doi.org/10.1093/heapol/14.4.374

Burke, T., McKee, J., Wilson, H., Donahue, R., Batenhorst, A., \& Pathak, D. (2000). A comparison of time-and-motion and self-reporting methods of work measurement. Journal of Nursing Administration, 30(3), 118-125. DOI: http://dx.doi. org/10.1097/00005110-200003000-00003

Bruland, P., Forster, C., Breil, B., Ständer, S., Dugas, M., \& Fritz, F. (2014). Does single-source create an added value? Evaluating the impact of introducing $\mathrm{X} 4 \mathrm{~T}$ into the clinical routine on workflow modifications, data quality and cost-benefit. International journal of medical informatics, 83(12), 915-928. DOI: http://dx.doi.org/10.1016/j.ijmedinf.2014.08.007

Cady, R., \&amp; Finkelstein, S. (2014). Task-Technology Fit of Video Telehealth for Nurses in an Outpatient Clinic Setting. Telemedicine and e- Health, 20(7), 633-639. DOI: http://dx.doi. org/10.1089/tmj.2013.0242

Carreira, X., Mariño, R., Perez-Cancio, X., \&amp; Fernández, M. (2013). Design of algorithms for evaluating the efficiency of labour and variability of some group milking parlour parameters. Livestock Science, 151(2), 252- 263. DOI: http:// dx.doi.org/10.1016/j.livsci.2012.11.012

Caso, A. (2006). Técnicas de medición del trabajo. FC Editorial. Ed, 2, 215. Chase, R., Jacobs, F. \&amp; Aquilano, N. (2009).

Administración de Operaciones producción y cadena de suministros. Editorial Mc Graw Hill, Ed. Duodécima.

Chisholm, C., Collison, E., Nelson, D., \&amp; Cordell, W. (2000). Emergency Department Workplace Interruptions Are Emergency Physicians "Interrupt-driven" and "Multitasking"?. Academic Emergency Medicine, 7(11), 1239-1243. DOI: http://dx.doi.org/10.1111/j.1553-2712.2000. tb00469.x
Chu, K., \&amp; Huang, C. (2013). Incremental analysis of the reengineering of an outpatient billing process: an empirical study in a public hospital. BMC health services research, 13(1),1. DOI: http://dx.doi.org/10.1186/1472-6963-13-215

Day, L., Belson, D., Dessouky, M., Hawkins, C., \&amp; Hogan, M. (2014). Optimizing efficiency and operations at a California safety-net endoscopy center: a modeling and simulation approach. Gastrointestinal endoscopy, 80(5), 762-773. DOI: http://dx.doi.org/10.1016/j. gie.2014.02.1032

Davis, A., Elkeeb, A., Vizzeri, G., \& Godley, B. (2016). Utilization of Portable Radios to Improve Ophthalmology Clinic Efficiency in an Academic Setting. Journal of medical systems, 40(3), 1-4. DOI: http://dx.doi.org/10.1007/s10916-0150417-x

De Coster, I., Fournie, X., Faure, C., Ziani, E., Nicolas, L., Soubeyrand, B., \& Van Damme, P. (2015). Assessment of preparation time with fully-liquid versus non-fully liquid paediatric hexavalent vaccines. A time and motion study. Vaccine, 33(32), 3976-3982. DOI: http://dx.doi. org/10.1016/j.vaccine.2015.06.030

De Cock, E., Kritikou, P., Ravera, S., \& Filippini, A. (2015). Time savings with once-monthly CERA: a time and motion study conducted in 13 haemodialysis centres in Italy. Blood purification, 40(2), 173-179. DOI: http://dx.doi. org/10.1159/000437133

Denton, G., Durning, S., Hemmer, P., \& Pangaro, L. (2005). RESEARCH BASIC TO MEDICAL EDUCATION: A Time and Motion Study of the Effect of Ambulatory Medical Students on the Duration of General Internal Medicine Clinics. Teaching and learning in medicine, 17(3), 285-289. DOI: http:// dx.doi.org/10.1207/s15328015t/m1703_15

Deo, S., Topp, S., Garcia, A., Soldner, M., Sokat, K., Chipukuma, J., ... \& Swann, J. (2012). Modeling the impact of integrating HIV and outpatient health services on patient waiting times in an urban health clinic in Zambia.

Rev. 12+D. Vol.16 N². Julio-Diciembre. 2016. Sogamoso-Boyacá, Colombia. 
PLoS One, 7(4), e35479. DOI: http://dx.doi. org/10.1371/journal.pone.0035479

Durán, F. (2007). Ingeniería de Métodos Globalización: Técnicas para el Manejo Eficiente de Recursos en Organizaciones Fabriles de servicios Hospitalarios. Premio Universal de Guayaquil.

Dwibedi, N., Sansgiry, S., Frost, C., Dasgupta, A., Jacob, S., Tipton, J. A., \& Shippy, A. (2011). Effect of bar-code-assisted medication administration on nurses' activities in an intensive care unit: A time-motion study. American Journal of Health-System Pharmacy, 68(11). DOI: http://dx. doi.org/10.2146/ajhp100382

Finkler, S., Knickman, J., Hendrickson, G., Lipkin Jr, M., \& Thompson, W. (1993). A comparison of work-sampling and time-and-motion techniques for studies in health services research. Health services research, 28(5), 577.

Fletcher, K., Visotcky, A., Slagle, J., Tarima, S., Weinger, M., \& Schapira, M.(2012). The composition of intern work while on call. Journal of general internal medicine, 27(11), 1432-1437. DOI: http://dx.doi.org/10.1007/s11606-012-2120-7

García , R. (2005). Estudio del trabajo: ingeniería de métodos y medición del trabajo. Editorial McGraw Hill Interamericana. México.

Groover, M. (2007). Work systems and the methods, measurement, and management of work (pp. 778-p). Upper Saddle River, NJ: Pearson Prentice Hall.

Hendrich, A., Chow, M., Skierczynski, B., \& Lu, Z. (2009). A 36-hospital time and motion study: how do medical-surgical nurses spend their time? RCHE Publications, 50.

Hodson K, W. (1996). MAYNARD, Manual del Ingeniero Industrial Tomo I. México, DF: editorial McGrawHill.

Hollingworth, W., Devine, E., Hansen, R., Lawless, N., Comstock, B., Wilson-Norton, J., ... \& Sullivan, S.(2007). The impact of e-prescribing on pres- criber and staff time in ambulatory care clinics: a time-motion study. Journal of the American Medical Informatics Association, 14(6), 722730. DOI: http://dx.doi.org/10.1197/jamia. M2377

Hontelez, J., Newell, M., Bland, R., Munnelly, K., Lessells, R., \& Bärnighausen, T. (2012). Human resources needs for universal access to antiretroviral therapy in South Africa: a time and motion study. Human resources for health, 10(1), 1. DOI: http://dx.doi.org/10.1186/1478-4491-10-39

Huang, E., Wang, H., Ko, P., Chang, A., Fu, C., Chen, J. \& Chiang, W. (2013). Obstacles delaying the prompt deployment of piston-type mechanical cardiopulmonary resuscitation devices during emergency department resuscitation: A video-recording and time-motion study. Resuscitation, 84(9), 1208-1213. DOI: http://dx.doi. org/10.1016/j.resuscitation.2013.03.028

Izetbegović, J. (2007). Work Study in Construction Industry.

Kanawaty, G. (1996). Introducción al estudio del trabajo. Ginebra, Organización internacional del Trabajo

Karger, D., \& Bayha, F. (1987). Engineered work measurement: the principles, techniques, and data of methods-time measurement background and foundations of work measurement and methods-time measurement, plus other related material. Industrial Press Inc.

Kee, R., Knott, J., Dreyfus, S., Lederman, R., Milton, S., \& Joe, K. (2012). One hundred tasks an hour: an observational study of emergency department consultant activities. Emergency Medicine Australasia, 24(3), 294-302. DOI: http://dx.doi.org/10.1111/j.1742-6723.2012.01540.x

Kreulen, C., Moscovich, H., Dansen, K., \& Creugers, N. (2000). Time-and-motion study on Class II copy-milled ceramic inlays. Journal of dentistry, 28(6), 429-436. DOI: http://dx.doi. org/10.1016/S0300-5712(00)00008-7 
Kilpatrick, K. (2013). How do nurse practitioners in acute care affect perceptions of team effectiveness?. Journal of clinical nursing, 22(17-18), 2636-2647. DOI: http://dx.doi.org/10.1111/ jocn. 12198

Kukafka, R., Allegrante, J., Khan, S., Bigger, J., \& Johnson, S.(2013). Understanding facilitators and barriers to reengineering the clinical research enterprise in community-based practice settings. Contemporary clinical trials, 36(1), 166-174. DOI: http://dx.doi.org/10.1016/j.cct.2013.06.008

Leafloor, C., Lochnan, H., Code, C., Keely, E., Rothwell, D., Forster, A., \& Huang, A.(2015). Time-motion studies of internal medicine residents' duty hours: a systematic review and meta-analysis. Advances in medical education and practice, 6, 621.

Li, L., Hains, I., Hordern, T., Milliss, D., Raper, R., \& Westbrook, J. (2015). What do ICU doctors do?: A multisite time and motion study of the clinical work patterns of registrars. Critical Care and Resuscitation, 17(3), 159.

Lo, H., Newmark, L., Yoon, C., Volk, L., Carlson, V., Kittler, A., ... \& Bates, D.(2007). Electronic health records in specialty care: a time-motion study. Journal of the American Medical Informatics Association, 14(5), 609-615. DOI: http:// dx.doi.org/10.1197/jamia.M2318

Lopetegui, M., Yen, P. Y., Lai, A., Jeffries, J., Embi, P., \& Payne, P. (2014). Time motion studies in healthcare: What are we talking about?. Journal of biomedical informatics, 49, 292-299. DOI: http://dx.doi.org/10.1016/j.jbi.2014.02.017

Lopez, K., Gerling, G., Cary, M., \& Kanak, M.(2010). Cognitive work analysis to evaluate the problem of patient falls in an inpatient setting. Journal of the American Medical Informatics Association, 17(3), 313-321. DOI: http://dx.doi.org/10.1136/ jamia.2009.000422

Lee, P., \& Chan, A. (2003). Chinese speaking times. International journal of industrial ergonomics, 31(5),
313-321. DOI: http://dx.doi.org/10.1016/S01698141(02)00222-6

MacMillan, T., Slessarev, M., \& Etchells, E. (2014). eWasted time: Redundant work during hospital admission and discharge. Health informatics journal, 1460458214534091.

Mache, S., Vitzthum, K., Klapp, B., \& Groneberg, D. (2012). Doctors' working conditions in emergency care units in Germany: a real-time assessment. Emergency Medicine Journal, 29(12), e1-e1. DOI: http://dx.doi.org/10.1136/emermed-2011-200599

Magu, P., Khanna, K., \& Seetharaman, P. (2015). Path Process Chart-A Technique for Conducting Time and Motion Study. Procedia Manufacturing, 3, 6475-6482. DOI.http://dx.doi.org/10.1016/j. promfg.2015.07.929

Mallidou, A., Cummings, G., Schalm, C., \& Estabrooks, C. (2013). Health care aides use of time in a residential long-term care unit: a time and motion study. International journal of nursing studies, 50(9), 1229-1239. DOI.http://dx.doi. org/10.1016/j.ijnurstu.2012.12.009

Mamykina, L., Vawdrey, D., Stetson, P., Zheng, K., \& Hripcsak, G. (2012). Clinical documentation: composition or synthesis?. Journal of the American Medical Informatics Association, 19(6), 1025-1031. DOI: http://dx.doi.org/10.1136/ amiajnl-2012-000901

Mangham-Jefferies, L., Mathewos, B., Russell, J., \& Bekele, A. (2014). How do health extension workers in Ethiopia allocate their time?. Human resources for health, 12(1), 1. DOI: http://dx.doi. org/10.1186/1478-4491-12-61

Manzi, F., Schellenberg, J., Hutton, G., Wyss, K., Mbuya, C., Shirima, K., ... \& Schellenberg, D. (2012). Human resources for health care delivery in Tanzania: a multifaceted problem. Human resources for health, 10(1), 1. DOI: http://dx.doi. org/10.1186/1478-4491-10-3 
Meguerditchian, A., Krotneva, S., Reidel, K., Huang, A., \& Tamblyn, R. (2013). Medication reconciliation at admission and discharge: a time and motion study. BMC health services research, 13(1), 1.DOI: http://dx.doi.org/10.1186/1472-6963-13485

Mensah, N., Sukums, F., Awine, T., Meid, A., Williams, J., Akweongo, P., ... \& Blank, A. (2015). Impact of an electronic clinical decision support system on workflow in antenatal care: the QUALMAT eCDSS in rural health care facilities in Ghana and Tanzania. Global ealth action, 8. DOI: http://dx.doi.org/10.3402/gha.v8.25756

Meyer, F. (2005). Estudio de Tiempos y Movimientos para la Manufactura Ágil, 4ta. Edición, México, Editorial Pearson Educación.

Milosavljevic, M., Williams, P., Perez, G., \& Dalla, T. (2011). The results of a pilot time-and-motion study in three Australian hospitals: Where do we spend our time?. Nutrition \& Dietetics, 68(3), 185-188. DOI: http://dx.doi. org/10.1111/j.1747-0080.2011.01526.x

Mizuyama, H., Yamada, K., Tanaka, K., \& Maki, A. (2013). Explanatory analysis of the manner in which an instructor adaptively organizes skilled motion teaching process. International Journal of Industrial Ergonomics, 43(5), 430-438. DOI. http://dx.doi.org/10.1016/j.ergon.2013.07.002

Murphy, D., Reis, B., Sittig, D., \& Singh, H. (2012). Notifications received by primary care practitioners in electronic health records: a taxonomy and time analysis. The American journal of medicine, 125(2), 209-e1. DOI: http://dx.doi. org/10.1016/j.amjmed.2011.07.029

Nakayama, S., Nakayama, K., \& Nakayama, H. (2002). A study on setting standard time using work achievement quotient. International journal of production research, 40(15), 3945-3953. DOI: http:// dx.doi.org/10.1080/00207540210159581

Niebel, B., Freivalds, A., \& Osuna, M. (2004). Ingeniería Industrial: Métodos, estándares y diseño del trabajo. Alfaomega. Ed Undecima.
Nor D,. (2008). Time Study Method Implementation in Manufacturing Industry.

Odendaal, W., \& Lewin, S. (2014). The provision of TB and HIV/AIDS treatment support by lay health workers in South Africa: a time-and-motion study. Human resources for health, 12(1), 1. DOI: http://dx.doi.org/10.1186/1478-4491-1218

Overhage, J., Perkins, S., Tierney, W., \& McDonald, C. (2001). Controlled trial of direct physician order entry: effects on physicians' time utilization in ambulatory primary care internal medicine practices. Journal of the American Medical Informatics Association, 8(4), 361-371. DOI: http:// dx.doi.org/10.1136/jamia.2001.0080361

Philpott, J., Shiferaw, S., Rouleau, K., Cole, D., Nicolle, E., Bezanson, K., ... \& Derbew, M. (2013). Family Medicine needs assessment: Studying the clinical work of general practitioners in Ethiopia. Ethiopian Journal of Health Development, 27(2), 134-140.

Pizziferri, L., Kittler, A., Volk, L., Honour, M., Gupta, S., Wang, S., ... \& Bates, D.(2005). Primary care physician time utilization before and after implementation of an electronic health record: a time-motion study. Journal of biomedical informatics, 38(3), 176-188. DOI: http://dx.doi. org/10.1016/j.jbi.2004.11.009

Poissant, L., Pereira, J., Tamblyn, R., \& Kawasumi, Y. (2005). The impact of electronic health records on time efficiency of physicians and nurses: a systematic review. Journal of the American Medical Informatics Association, 12(5), 505-516. DOI: http://dx.doi.org/10.1197/jamia.M1700

Poon, E., Keohane, C., Bane, A., Featherstone, E., Hays, B., Dervan, A., ... \& Gandhi, T.(2008). Impact of barcode medication administration technology on how nurses spend their time providing patient care. Journal of Nursing Administration, 38(12), 541-549. DOI: http://dx.doi. org/10.1097/NNA.0b013e31818ebf1c 
Prenner, J., Halperin, L., Rycroft, C., Hogue, S., Liu, Z., \& Seibert, R. (2015). Disease burden in the treatment of age-related macular degeneration: findings from a time-and-motion study. American journal of ophthalmology, 160(4), 725-731. DOI: http://dx.doi.org/10.1016/j.ajo.2015.06.023

Puvanasvaran, A., Mei, C., \& Alagendran, V. (2013). Overall equipment efficiency improvement using time study in an aerospace industry. Procedia Engineering, 68, 271-277. DOI: http://dx.doi. org/10.1016/j.proeng.2013.12.179

Qian, S., Yu, P., Zhang, Z., Hailey, D., Davy, P., \& Nelson, M. (2012). The work pattern of personal care workers in two Australian nursing homes: a time-motion study. BMC health services research, 12(1), 1. DOI: http://dx.doi. org/10.1186/1472-6963-12-305

Qian, S., Yu, P., Hailey, D., Zhang, Z., Davy, P., \& Nelson, M. (2014). Time spent on daytime direct care activities by personal carers in two Australian residential aged care facilities: a time-motion study. Australian Health Review, 38(2), 230237. DOI: http://dx.doi.org/10.1071/ah13161

Quach, S., Hamid, J., Pereira, J., Heidebrecht, C., Foisy, J., Bettinger, J., ... \& Finkelstein, M. (2011). Time and motion study to compare electronic and hybrid data collection systems during the pandemic (H1N1) 2009 influenza vaccination campaign. Vaccine, 29(10), 1997-2003 DOI: http:// dx.doi.org/10.1016/j.vaccine.2010.09.016

Rebmann, T., Clements, B., Bailey, J., \& Evans, R.(2009). Organophosphate antidote auto-injectors vs. traditional administration: a time motion study. The Journal of emergency medicine, 37(2), 139143. DOI: http://dx.doi.org/10.1016/j.jemermed.2007.09.043

Robledo, S., Osorio, G., \& Lopez, C. (2015). Networking en pequeña empresa: una revisión bibliográfica utilizando la teoría de grafos. Vínculos, 11(2), 6-16.

Roman, C., Poole, S., Walker, C., \& Dooley, M. (2016). A 'time and motion'evaluation of automated dispensing machines in the emergency department. Australasian Emergency Nursing Journal, 19(2), 112-117. DOI: http://dx.doi.org/10.1016/j.aenj.2016.01.004

Rothberg, M., Steele, J., Wheeler, J., Arora, A., Priya, A., \& Lindenauer, P.(2012). The relationship between time spent communicating and communication outcomes on a hospital medicine service. Journal of general internal medicine, 27(2), 185-189. DOI: http://dx.doi.org/10.1007/ s11606-011-1857-8

Rudkin, S., Langdorf, M., Macias, D., Oman, J., \& Kazzi, A.(2006). Personal digital assistants change management more often than paper texts and foster patient confidence. European Journal of Emergency Medicine, 13(2), 92-96. DOI: http://dx.doi. org/10.1097/01.mej.0000192049.04729.0c

Rudkin, S., \& O'Donoghue, P.(2008). Time-motion analysis of first-class cricket fielding. Journal of Science and Medicine in Sport, 11(6), 604-607. DOI: http://dx.doi.org/10.1016/j.jsams.2007.08.004

Russell, R., \& Taylor, B.(2003). Operations management. Vol.3. Jersey New Jersey: Prentice Hall.

Saurman, E., Lyle, D., Kirby, S., \& Roberts, R. (2014). Assessing Program Efficiency: A Time and Motion Study of the Mental Health Emergency CareRural Access Program in NSW Australia. International journal of environmental research and public health, 11(8), 7678-7689. DOI: http:// dx.doi.org/10.3390/ijerph110807678

Schofield, B., Cresswel, K., Westbrook, J., Slee, A., Girling, A., Shah, S., ... \& Sheikh, A. (2015). The impact of electronic prescribing systems on pharmacists' time and workflow: protocol for a time-and-motion study in English NHS hospitals. BMJ open, 5(10), e008785. DOI: http://dx. doi.org/10.1136/bmjopen-2015-008785

Shabbir, S., Ahmed, L., Sudhir, R., Scholl, J., Li, Y., \& Liou, D. (2010). Comparison of documentation time between an electronic and a paper-based record system by optometrists at an eye hospital in south India: A time-motion

Rev. I2+D. Vol.16 N². Julio-Diciembre. 2016. Sogamoso-Boyacá, Colombia. 
study. Computer methods and programs in biomedicine, 100(3), 283-288. DOI: http://dx. doi.org/10.1016/j.cmpb.2010.04.003

Shinder, G., Paradis, P., Posman, M., Mishagina, N., Guay, M., Linardos, D., \& Batist, G. (2012). Patient and work flow and costs associated with staff time and facility usage at a comprehensive cancer centre in Quebec, Canada-a time and motion study. BMC health services research, 12(1), 1. DOI: http://dx.doi.org/10.1186/1472$6963-12-370$

Spinelli, R., \& Visser, R.(2009). Analyzing and estimating delays in wood chipping operations. Biomass and bioenergy, 33(3), 429-433. DOI: http://dx. doi.org/10.1016/j.biombioe.2008.08.003

Starmer, A., Destino, L., Yoon, C., \& Landrigan, C. (2015). Intern and Resident Workflow Patterns on Pediatric Inpatient Units: A Multicenter Time-Motion Study. JAMA pediatrics, 169(12), 1175-1177. DOI: http://dx.doi.org/10.1001/jamapediatrics.2015.2471

Suh, D., Powers, C., Barone, J., Shin, H., Kwon, J., \& Goodin, S. (2010). Full costs of dispensing and administering fluorouracil chemotherapy for outpatients: A microcosting study. Research in Social and Administrative Pharmacy, 6(3), 246-256. DOI: http://dx.doi.org/10.1016/j.sapharm.2009.07.004

Tanner, J. (1990). Manufacturing Engineering: An Introduction to the Basic Functions, Revised and expanded (Vol. 36). CRC Press.

Tinoco, H., Ovalle, A., Vargas, C., \& Cardona, M. (2015). An automated time and hand motion analysis based on planar motion capture extended to a virtual environment. Journal of Industrial Engineering International, 11(3), 391-402. DOI: http://dx.doi.org/10.1007/s40092-015-0107-9

Tipping, M., Forth, V., O'Leary, K., Malkenson, D. M., Magill, D., Englert, K., \& Williams, M. (2010). Where did the day go?-A time-motion study of hospitalists. Journal of hospital medicine, 5(6), 323328. DOI: http://dx.doi.org/10.1002/jhm.790
Thorpe-Jamison, P., Culley, C., Perera, S., \& Handler, S. (2013). Evaluating the impact of computer-generated rounding reports on physician workflow in the nursing home: A feasibility time-motion study. Journal of the American Medical Directors Association, 14(5), 358-362. DOI: http:// dx.doi.org/10.1016/j.jamda.2012.11.008

Torres, P; Perez,A; Marmolejo,L; Ordoñez,J ; Garcia,R (2010) Una mirada a la agroindustria de extracción de almidón de yuca,desde la estandarización de procesos.RevistaEIA,Nùmero 14,p.2338

Tuinman, A., de Greef, M., Krijnen, W., Nieweg, R., \& Roodbol, P. (2015). Examining Time Use of Dutch Nursing Staff in Long-Term Institutional Care: A Time-Motion Study. Journal of the American Medical Directors Association, 17(2), 148-154. DOI: http://dx.doi.org/10.1016/j.jamda.2015.09.002

Turner, W. (1993). Introduction to industrial and systems engineering. New Jersey. Pearson College Division.

van Oostveen, C., Vermeulen, H., Gouma, D., Bakker, P., \& Ubbink, D.(2013). Explaining the amount of care needed by hospitalised surgical patients: a prospective time and motion study. BMC health services research, 13(1), 1. DOI: http://dx.doi. org/10.1186/1472-6963-13-42

Van Oostveen, C., Gouma, D., Bakker, P., \& Ubbink, D. (2015). Quantifying the demand for hospital care services: a time and motion study. $B M C$ health services research, 15(1), 1. DOI: http:// dx.doi.org/10.1186/s12913-014-0674-2

Victores, A., Coggins, K., \& Takashima, M. (2015). Electronic health records and resident workflow: A time-motion study of otolaryngology residents. The Laryngoscope, 125(3), 594-598. DOI: http://dx.doi.org/10.1002/lary.24848

Vonderembse, M., \& White, G. (2003). Core concepts of operations management. John Wiley \& Sons Inc. 
Wanyenze, R., Wagner, G., Alamo, S., Amanyire, G., Ouma, J., Kwarisima, D., ... \& Kamya, M. (2010). Evaluation of the efficiency of patient flow at three HIV clinics in Uganda. AIDS patient care and STDs, 24(7), 441-446. DOI: http://dx.doi. org/10.1089/apc.2009.0328

Weigl, M., Müller, A., Zupanc, A., \& Angerer, P. (2009). Participant observation of time allocation, direct patient contact and simultaneous activities in hospital physicians. BMC health services research, 9(1), 1. DOI: http://dx.doi. org/10.1186/1472-6963-9-110

Weigl, M., Müller, A., Sevdalis, N., \& Angerer, P. (2013). Relationships of multitasking, physicians' strain, and performance: an observational study in ward physicians. Journal of patient safety, 9(1), 18-23.

Were, M., Emenyonu, N., Achieng, M., Shen, C., Ssali, J., Masaba, J., \& Tierney, W. (2010). Evaluating a scalable model for implementing electronic health records in resource-limited settings. Journal of the American Medical Informatics Association, 17(3), 237-244. DOI: http:// dx.doi.org/10.1136/jamia.2009.002303

Westbrook, J., \& Ampt, A. (2009).Design, application and testing of the Work Observation Method by Activity Timing (WOMBAT) to measure clinicians' patterns of work and communication. International journal of medical informatics, 78, S25-S33. DOI: http://dx.doi.org/10.1016/j.ijmedinf.2008.09.003

Westbrook, J., Coiera, E., Dunsmuir, W., Brown, B., Kelk, N., Paoloni, R., \& Tran, C. (2010). The impact of interruptions on clinical task completion. Quality and Safety in Health Care, 19(4), 284-289. DOI: http://dx.doi.org/10.1136/ qshc. 2009.039255

Westbrook, J., Li, L., Georgiou, A., Paoloni, R., \& Cullen, J. (2013). Impact of an electronic medication management system on hospital doctors' and nurses' work: a controlled pre-post, time and motion study. Journal of the American Medical Infor- matics Association, 20(6), 1150-1158 DOI: http:// dx.doi.org/10.1136/amiajnl-2012-001414

Wiedenmayer, K., Weiss, S., Chattopadhyay, C., Mukherjee, A., Kundu, R., Ayé, R., ... \& Tanner, M. (2009). Simplifying paediatric immunization with a fully liquid DTP-HepB-Hib combination vaccine: Evidence from a comparative time-motion study in India. Vaccine, 27(5), 655-659. DOI: http:// dx.doi.org/10.1016/j.vaccine.2008.11.045

Wong, R., \& Bradley, E. (2009). Developing patient registration and medical records management system in Ethiopia. International Journal for Quality in Health Care, 21(4), 253-258. DOI: http:// dx.doi.org/10.1093/intqhc/mzp026

Wygant, R. (1989). A comparison of computerized predetermined time systems. Computers \& industrial engineering, 17(1), 480-485. DOI: http:// dx.doi.org/10.1016/0360-8352(89)90109-5

Shuang, W,. Wang, Y., BolaBola, J., Qin, H., Ding, W., Wen, W., \& Niu, J. (2016). Incorporating motion analysis technology into modular arrangement of predetermined time standard (MODAPTS). International Journal of Industrial Ergonomics, 53, 291-298. DOI: http://dx.doi. org/10.1016/j.ergon.2016.03.001

Yusoff, N., Jaffar, A., Abbas, N., \& Saad, N. (2012). Work measurement for process improvement in the car seat polyurethane injection manufacturing line. Procedia Engineering, 41, 1800-1805. DOI: http://dx.doi.org/10.1016/j. proeng.2012.07.386

Zheng, K., Guo, M., \& Hanauer, D. (2011). Using the time and motion method to study clinical work processes and workflow: methodological inconsistencies and a call for standardized research. Journal of the American Medical Informatics Association, 18(5), 704-710. DOI: http://dx.doi. org/10.1136/amiajnl-2011-000083.

Rev. I2+D. Vol.16 N². Julio-Diciembre. 2016. Sogamoso-Boyacá, Colombia. 